\title{
Fokus auf die \\ Medikationssicherheit
}

\author{
Olga Frank ${ }^{a}$, Petra Seeburger ${ }^{b}$ \\ ${ }^{a}$ Dr., Projektleitung, Patientensicherheit Schweiz; ${ }^{b}$ Leitung Kommunikation, Patientensicherheit Schweiz
}

\section{Zusammenfassung}

Patientensicherheit Schweiz ist es ein grosses Anliegen, allen an der medizinischen Behandlung beteiligten Gesundheitsinstitutionen im Rahmen einer Aktionswoche eine nationale Plattform zu bieten, mittels der sie aufzeigen können, wie und was sie alles zur Förderung der Patientensicherheit tun. Zum zweiten Mal

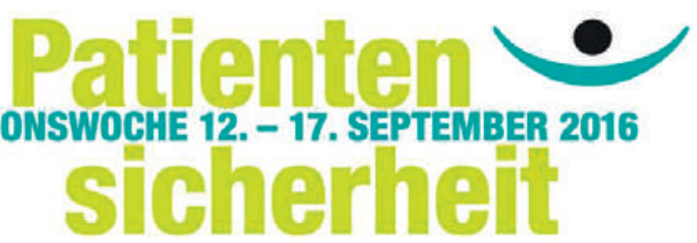

führt die Stiftung in diesem Jahr eine Aktionswoche zur Patientensicherheit durch und ruft zur Beteiligung auf. Im Fokus der Aktivitäten steht das Thema der «Medikationssicherheit» als ein wichtiger Hot-Spot der Patientensicherheit.

\section{Aktionswoche Patientensicherheit 2016}

Die Patientensicherheit ist aus dem Berufsalltag der Ärztinnen und Ärzte nicht wegzudenken. Sie ist die Grundlage einer guten und patientenzentrierten Gesundheitsversorgung und von allgemeinem und gesellschaftlichem Interesse. Sie gehört zu den fundamentalsten Qualitätsmerkmalen einer medizinischen Behandlung und ist deshalb eine zentrale Aufgabe aller Akteure im Gesundheitswesen. Im Hinblick auf die Sicherstellung einer fehlerfreien medizinischen Behandlung wird in den Schweizer Gesundheitsinstitutionen bereits sehr viel geleistet. Dennoch muss noch viel getan werden, damit alle an der medizinischen Behandlung beteiligten Player an einem Strang ziehen. So ist es auch wichtig, die Öffentlichkeit zu sensibilisieren und zu informieren sowie bestehende Anstrengungen zur Förderung der Patientensicherheit sichtbar zu machen. Patientensicherheit Schweiz führt vom 12. bis 17. September 2016 zum zweiten Mal eine natio- nale Aktionswoche durch. Nach dem grossen Erfolg der ersten Aktionswoche im vergangenen Jahr und den positiven Evaluationsergebnissen soll wiederholt allen Leistungserbringern die Möglichkeit geboten werden, ihre Anstrengungen zur Förderung der Patientensicherheit aufzuzeigen und gemeinsam mit anderen Playern konzentriert in einer Woche der Öffentlichkeit zu präsentieren.

\section{Fokusthema Medikationssicherheit}

Unerwünschte Arzneimittelwirkungen und Medikationsfehler gehören zu den grössten Risiken, welche die Patientensicherheit im Spital beeinträchtigen. Geschätzte 20000 Spitalaufenthalte pro Jahr sind in der Schweiz auf medikamentenbedingte Probleme zurückzuführen ${ }^{1}$. Ein Drittel dieser Spitalaufenthalte wäre vermeidbar'. Jeder zwanzigste befragte Schweizer gab in einer nationalen Erhebung an, innerhalb der letzten zwei Jahre mindestens einmal ein falsches Medikament oder eine falsche Dosis erhalten zu haben ${ }^{2}$. Medikationssicherheit muss durch Massnahmen im gesamten Medikationsprozess optimiert werden. Die Medikationssicherheit an Schnittstellen, also an Behandlungsübergängen, ist besonders fehleranfällig und steht deshalb im Zentrum des nationalen Programms progress! Sichere Medikation an Schnittstellen der Stiftung Patientensicherheit Schweiz. Die meisten Patienten haben beim Verlassen des Spitals eine andere Arzneimitteltherapie als bei Spitaleintritt. Bei jedem zweiten Eintritt in eine Klinik kommt es zu Unstimmigkeiten. Durch systematische Kontrollen der Medikamentenpläne lassen sich Medikationsfehler vermeiden.

Die Medikationssicherheit, als ein wichtiger Hot-Spot in der Patientensicherheit, steht deshalb auch im Fokus der diesjährigen Aktionswoche und des internationalen Tags der Patientensicherheit am 17. September 2016, an dem sich die deutschen und österreichischen Patientensicherheits-Organisationen, evtl. auch ähnlich gelagerte Institutionen aus den umliegenden europäischen Ländern, beteiligen werden. 


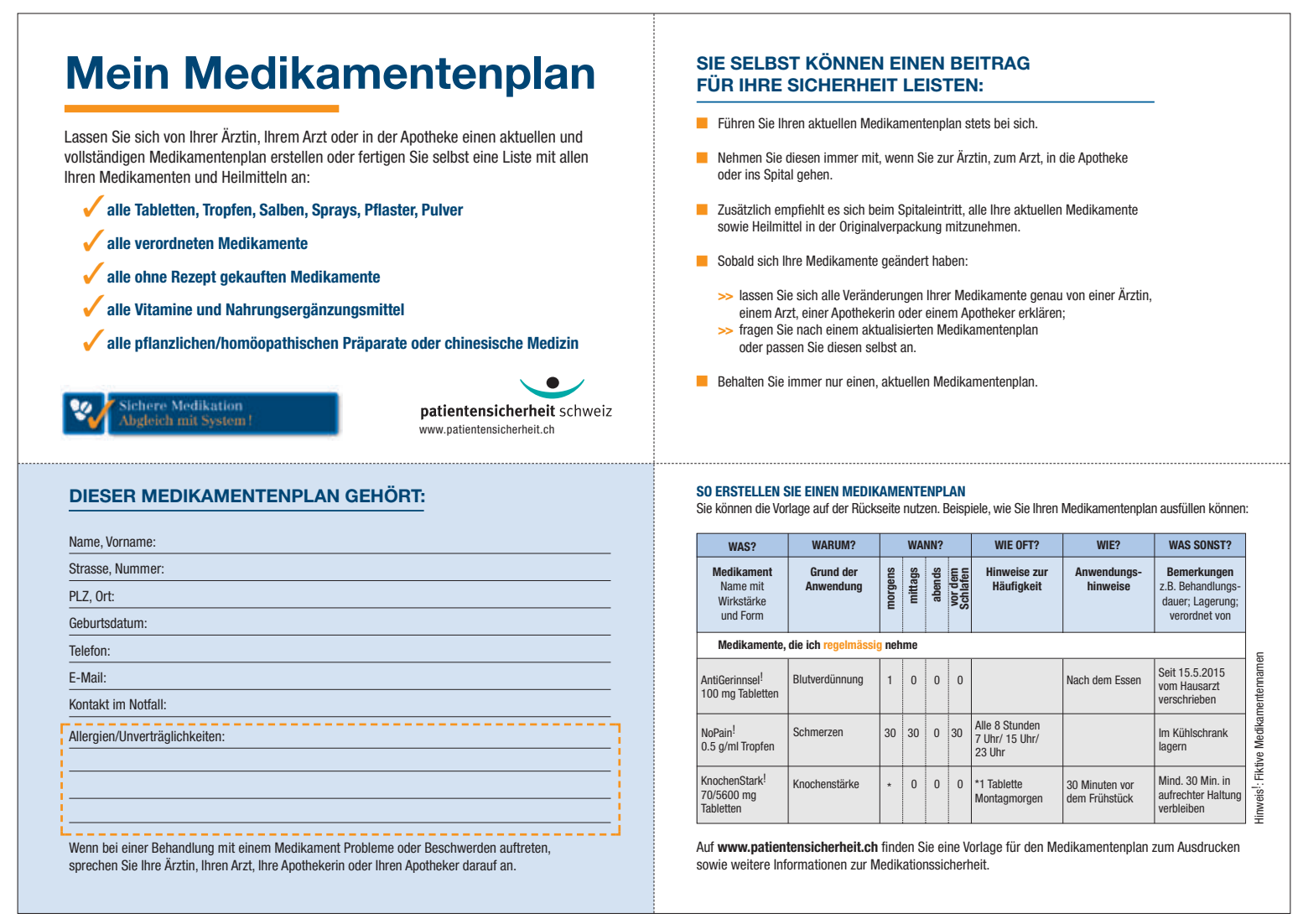

Abbildung 1: Medikamentenplan.

\section{Aktivitäten der Gesundheitsinstitutionen}

Wie im letzten Jahr sind Aktivitäten erwünscht, die einen direkten Bezug zur Patientensicherheit haben: Posterausstellungen, Symposien, Tag der offenen Tür, Veranstaltungen für Mitarbeitende und Patienten, Standaktionen und anderes.

Patientensicherheit Schweiz wird vor 16 Spitälern einen Medikamenten-Bag mit einem Medikamentenplan an Patienten und Besucher abgeben. Der Medikamentenplan wurde im Rahmen des nationalen Programms progress! Sichere Medikation an Schnittstellen entwickelt und soll nun breite Anwendung finden. Durch die Unterstützung der Schweizerischen Gesellschaft für Allgemeine Innere Medizin (SGAIM), des Schweizerischen Vereins der Amts- und Spitalapotheker (GSASA) und der pharmaSuisse erhalten zudem alle öffentlichen Apotheken und alle Schweizer Hausärztinnen und Hausärzte Gratisexemplare dieses Medikamentenplans zugestellt.

\section{Aufruf zu Beteiligung}

Aufgerufen sind alle Gesundheitseinrichtungen der Schweiz! Beteiligen auch Sie sich mit einer Aktion zur
Patientensicherheit und nutzen Sie die Möglichkeit, sichtbar zu machen, was Ihre Institution für Massnahmen zur Förderung der Patientensicherheit unternimmt. Entscheidend ist nicht die Grösse der Aktion, sondern Ihr Engagement in Sachen Patientensicherheit. Unterstützende Materialien sind auf der Website der Aktionswoche downloadbar unter www. aktionswoche-patientensicherheit.ch.

Literatur

- Hardmeier B, Braunschweig S, Cavallaro M, Roos M, PauliMagnus C, Giger M, et al. Adverse drug events caused by medication errors in medical inpatients. Swiss Med Wkly 2004;134(45-46):664-70.

- Wasserfallen J, Livio F, Buclin T, Tillet L, Yersin B, Biollaz J. Rate, type, and cost of adverse drug reactions in emergency department admissions. European Journal of Internal Medicine 2001;12(5):442-7.

- Lepori V, Perren AF, Marone C. Unerwünschte internmedizinische Arzneimittelwirkungen bei Spitaleintritt [Adverse internal medicine drug effects at hospital admission]. Schweizerische Medizinische Wochenschrift 1999;129(24): 915-22.

- Schwappach DL. Risk factors for patient-reported medical errors in eleven countries. Health Expect 2012;17(3):321-31. 